\title{
Organic Hallucinosis in Non-Hemorrhagic Stroke Caused by Thrombosis Process: A Case Report
}

\author{
Pricella Maria Ismail ${ }^{1}$, Anak Ayu Sri Wahyuni' ${ }^{1}$, \\ Anak Agung Ayu Suryapraba Indradewi ${ }^{2}$
}

${ }^{1}$ Department of Psychiatry, Faculty of Medicine, Udayana University, Denpasar, Bali, Indonesia, 80113
${ }^{2}$ Department of Neurology, Faculty of Medicine, Udayana University, Denpasar, Bali, Indonesia, 80113

Corresponding Author: Anak Ayu Sri Wahyuni

DOI: https://doi.org/10.52403/ijrr.20220108

\begin{abstract}
Background/aim: Hallucinations are the special ability to experience phenomena that are not visible to normal individuals. Hallucinations, delusions, and confabulations are common symptoms between neurology and psychiatry. Nervous disease that manifests with hallucinatory symptoms like this is one of them due to right hemispheric stroke. The authors report cases of new-onset organic hallucinosis. and stroke in brain regions similar to the salience network (insular cortex, parietal cortex, and striatum).
\end{abstract}

Case: A 43-year-old man comes to the ER Sanglah Hospital Denpasar, Bali Indonesia with complaints of slurred speech using an incomprehensible language, and repeating the same words. Talking about seeing a shadow following him but actually not there. Patients often experience sleep disorders, from the results of neurological physical examination found right eye ptosis, pupil anisokor, nerve III dextra complete lesion, supranuclear left NVII paresis, supranuclear left NXII paresis, left flaccid hemiparesis. Psychiatric status obtained unnatural appearance, looks confused, verbal and visual contact is sufficient, mood dysphoric, confused affect and there is no harmony. The thought process obtained realistic, coherent, preoccupation with pain. Perceptual disturbances in the form of visual and auditory hallucinations. Insomnia mixed type and there is hypobulia. Psychomotor calm on examination, history increases. Narcissistic personality traits and defense mechanisms of ego repression. Grade 4 view. CT scan of the head with and without contrast shows subacute ischemic cerebral infarction in the right internal capsule to the right thalamus and midbrain

Conclusion: Organic hallucinosis occur in nonhemorrhagic stroke caused by thrombosis process if an infarction is found in the right hemisphere.

Keywords: organic hallucinations, ischemic cerebral infarction, non-hemorrhagic stroke, right hemisphere

\section{INTRODUCTION}

Hallucination has its foundations in the Latin hallucinari or allucinari, which signifies 'strolling in the brain'. Contemporary meaning of hallucination proposed by David in 2004 is that tactile encounters happen without outer improvements from the applicable tangible organs, have a reality that adequately looks like genuine discernment, which the subject doesn't see straightforwardly and happens in a conscious state. David makes a few significant focuses in his definition. To start with, mind flights are basically abstract tactile encounters and are in this manner fairly impervious to true investigation utilizing the logical technique. Second, the degree of understanding of individuals who experience mind flights have differing reality encounters, for instance a patient with schizophrenia might accept that the sounds they hear are coming from a recording device in the room and are off. Conversely, patients with Charles Bonnet Syndrome (CBS) frequently understand that 
the hallucinations are artificial. Third, in spite of the fact that individuals with hallucination are impacted somewhat by the hallucination state, they for the most part have zero command over themselves. At last, dreams are false hallucination since they don't happen while conscious.[1-3]

In antiquated occasions, there was an extremely current order of hallucinations. The Hippocratic school considered the experience of sight and sound to be identified with suspicion as recognized from epilepsy, madness and despairing. In this manner, the experience of sight and sound is delegated a unitary clinical encounter. Hippocrates accepted that hallucinations were the exceptional capacity to encounter peculiarities not seen by ordinary people. This kind of 'obtained work' clarification for hallucinations had a typical subject in old occasions, for instance a high Pythian cleric in the eighth century BC encountering hallucination that were deciphered as prediction. Pythagoras thought the mathematician was directed by strong. Indeed, even Socrates wondered about the apparent voice which he accepted was past his own brain, which had been alluded to as the Socratic Demon.[1,3,4]

Case of hallucination, delusions, and confabulations are common symptoms between neurology and psychiatry. Neurological sicknesses showing with manifestations, for example, dementia, epilepsy, Korsakoff's illness, cerebrum growths, Parkinson's infection, headache, right hemispheric stroke and others will be the way to understanding their organic components, while intellectual science, neuropharmacology and useful neuroimaging will be the devices of study. It is feasible to comprehend the principles of psyche insight and the systems of human awareness dependent on these peculiarities. In any case, mind flights and perception show with mental problems like psychosis and schizophrenia, for which there is no proof of cerebrum sores. Also, hallucination and dreams are emotional manifestations, and they have no natural markers. In this manner, high between individual fluctuation and ward on different factors (like training, history of injury), and hard to decrease to a firm system. The causal instruments might be many. To comprehend these indications, the overall structure among nervous system science and psychiatry remains.[5]

A stroke is brain damage that occurs suddenly caused by a failure of blood supply where there is a blood clot in one of the arteries or blood vessels of the brain that blocks circulation to certain areas of the brain or an artery ruptures (bleeds). The impact of stroke can cause cognitive and perceptual disturbances. The more common type of stroke is ischemia, the result of a blood clot or other obstruction in the artery. A rare type is haemorrhage, resulting from arterial rupture. Strokes vary in severity from barely noticeable to fatal. A stroke kills brain cells immediately, but those in the penumbra persist at least temporarily.[6] The authors report cases of new-onset organic hallucinosis and stroke in brain regions similar to the salience network (insular cortex, parietal cortex, and striatum).

\section{CASE REPORT}

A male, 48 years old, older than his age, long curly hair, messy, right eyelid closed compared to left eye, tall stature, long curly hair and looks confused. Patients interviewed using Indonesian, responded with a slurred voice and sentences that were not clearly understood.

He was consulted for leaving his room last night. The patient said that he saw an unknown man come riding a motorbike into his room and take him to ride a motorbike with him. The patient narrates the incident in a sentence that is not understood. When telling about last night's incident, the his screamed in pain in his anus and asked for an immediate operation for his "hemorrhoid".

His complained of "hemorrhoid" pain since 4 days ago, especially when defecating, a lump came out and it was difficult to put it back in the anus. The 
Pricella Maria Ismail et.al. Organic hallucinosis in non-hemorrhagic stroke caused by thrombosis process: a case report.

patient repeatedly said about the "hemorrhoid" pain, wanting surgery immediately so that he could go home quickly and feel more comfortable when he was at home. The patient did not think much about the weakness of his left body and the stroke he was experiencing. In addition to the image of the person he saw last night, the patient sometimes misunderstood his nephew, said the cleaning service and hospital nurses, so that his nephew repeated his name.

The patient's sleep was disturbed, where the patient was able to sleep at first, then woke up because of the pain for about 30 minutes until it disappeared, only to be able to go back to sleep every night for 4 days. Initially, the patient's appetite was normal and finally reduced for fear of not being able to excrete it. The patient is a person who has many friends, is famous, likes to hang out and hang out with friends, is active in all social activities, dares to approach important people in government. If there is a problem, the patient does not tell anyone, including the "hemorrhoid" pain.

On neurological examination found right eye ptosis, pupil anisokor, nerve III dextra complete lesion, supranuclear left NVII paresis, supranuclear left NXII paresis, left flaccid hemiparesis. On examination of the surgical status, a dark red perianal lump appeared. Psychiatric status obtained unnatural appearance, looks confused, verbal and visual contact is sufficient, mood dysphoric, confused affect and there is no harmony. The thought process obtained realistic, coherent, preoccupation with pain. Perceptual disturbances in the form of visual and auditory hallucinations. Insomnia of mixed type and there is hypobulia. Psychomotor calm on examination, history increases. Narcissistic personality traits and defense mechanisms of ego repression. 4th degree view.

In the patient, behavioral and psychological symptoms were found which were clinically quite significant and caused distress and disability in daily life which indicated that the patient had a mental disorder. CT scan of the head axial examination with and without contrast shows subacute ischemic cerebral infarction in the right internal capsule to the right thalamus and midbrain (Fig 1).

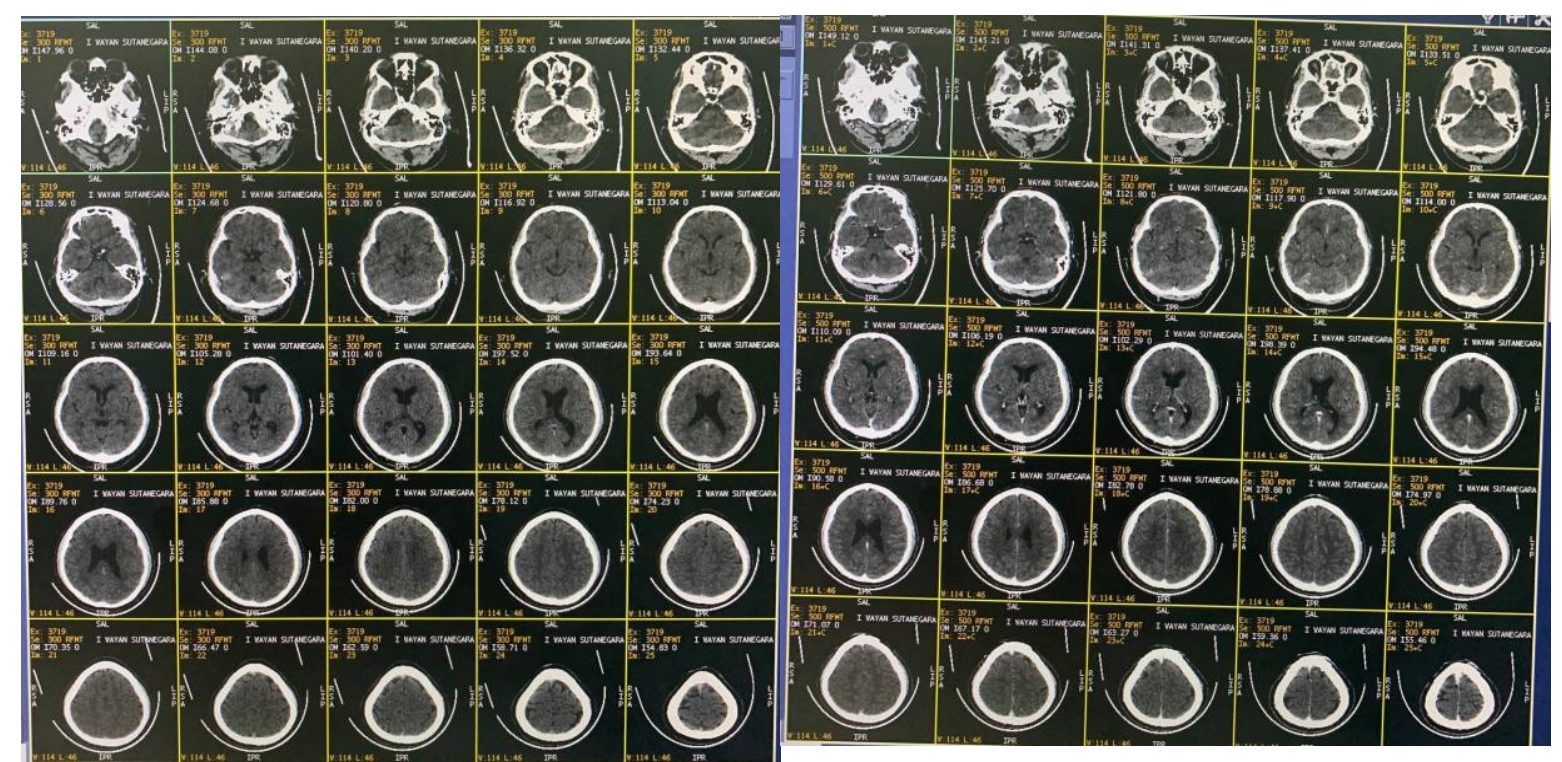

Figure 1. CT scan of the head axial examination with and without contrast shows subacute ischemic cerebral infarction in the right internal capsule to the right thalamus and midbrain

History taking and physical examination found general medical disorders that physiologically can cause brain dysfunction and cause mental disorders currently being suffered, namely Non- Hemorrhagic Stroke and Thrombus, so 
that there are Other Mental Disorders Due to Brain Damage and Dysfunction and Physical Disease (F06), namely Organic Hallucinosis can be established as an Axis 1 diagnosis cause:

1. Disease, brain damage or dysfunction, or systemic physical illness known to be associated with one of the listed mental syndromes.

2. There is a time relationship (in weeks or months) between the development of the underlying disease and the onset of a mental syndrome

3. Recovery from mental disorders after repair or elimination of the underlying cause

4. There is no evidence pointing to alternative causes for this mental syndrome (such as a strong influence from family history or the influence of stress as a trigger)

There was also no history of use of psychoactive substances and symptoms of dependence and withdrawal from the patient. The patient smokes 20 cigarettes a day, drinks 3 cups of coffee a day and rarely drinks alcohol. During illness, the patient does not look for cigarettes and there is a primary disease in the brain that precedes so that Mental and Behavioral Disorders Due to Psychoactive Substances used can be ruled out.

His does not experience impaired consciousness, sleep-wake cycle disturbances with rapid onset, fluctuating course of the disease throughout the day lasting less than 6 months despite experiencing hallucinations and several other sensory cognition disorders so that Delirium, not induced by alcohol and other psychoactive substances (F05) can be ruled out.

His experienced a perceptual disturbance, namely hallucinations that occurred less than 2 weeks but were preceded by brain disease. According to ICD10, Acute Schizophrenic-like psychotic disorder (F23.2) can be ruled out.

$\mathrm{He}$ is a person who has many friends, is famous, likes to hang out and hang out with friends, is active in all social activities, dares to approach important people in government. If there is a problem, the patient does not tell anyone including the "hemorrhoid" pain, so the Narcissistic personality trait with ego defense mechanism Repression.

He experiencing general medical conditions, namely Non-Hemorrhagic Stroke ec Thrombus; CHF FC II ec suspend HHD, HT stage II; Dyslipidemia; hyperuricemia; Grade IV internal hemorrhoids become Axis III.

He experiencing a condition where a Non-Hemorrhagic Stroke ec Thrombus; CHF FC II ec suspend HHD, HT stage II; Dyslipidemia; hyperuricemia; Internal hemorrhoids grade IV which causes disturbances in the field of psychiatry. In Axis IV, the problems with the disease faced by the patient were recorded as problems related to health conditions, namely Non-Hemorrhagic Stroke and Hemorrhoids.

Axis V Global Assessment of Functioning Scale (GAF) is currently assessed as 30-21 with severe disabilities in communication and judgment, unable to function in almost all areas. GAF for the past year was 90-81 where symptoms were minimal, functioning well, moderately satisfied, nothing more than normal daily problems. A "organobiological factors" there are Non-Hemorrhagic Stroke Ec Thrombus; CHF FC II ec suspend HHD, HT stage II; Dyslipidemia; hyperuricemia; Grade IV internal hemorrhoids are associated as the cause of the current mental disorder in the patient. The family experienced the same thing as the patient.

He have personality is a person who has many friends, is famous, likes to hang out and hang out with friends, is active in all social activities, dares to approach important people in government who shows Narcissistic Personality traits. According to the DSM-5, Narcissistic personality disorder meets the following criteria: A pervasive pattern of grandiosity (in hallucination or behavior) requiring pride and a lack of 
empathy, beginning in early adulthood and appearing in a variety of contexts, as indicated by five (or more) of the following:

1. A great sense of self-importance (e.g. excessive achievement and talent, wishing to be recognized as superior without commensurate effort).

2. Preoccupation with hallucination of infinite success, strength, intelligence, beauty or ideal love.

3. That it is "special" and unique and can be understood only by or having to relate to other people (or institutions) of special or high status.

4. Need too much pride

5. Having high feelings i.e. unwarranted expectations of special treatment or automatic compliance with expectations

6. Interpersonally exploitative i.e. taking advantage of others to achieve one's own goals

7. Do not have empathy, do not want to recognize or know the feelings and wholeness of others

8. Always feels jealous of others or believes that others are jealous of him

9. Showing arrogant behavior or attitude

He experienced a problem, he tended to always keep it to himself and rarely told his family, which is a defense mechanism for ego repression. Defense mechanism by avoiding the conflict that is faced without realizing it. One day this conflict that is stored in the subconscious will be able to surface and can interfere with his life. An idea or feeling can be removed or withheld from consciousness through repression. Primary repression is restraining ideas and feelings before they reach consciousness; secondary repression is removing from consciousness what has been experienced on a conscious level. The thing that is repressed is not completely forgotten, so that symbolic behavior can be discovered.

Psychological factors, childhood parenting, both parents divorced when they were young. His does not get enough attention and affection from both parents. According to Freud's psychosexual theory, patients may experience fixation in the Oral phase, which triggers excessive optimism, narcissism, pessimism and demands. Patients who receive less attention from their families and their outlets by seeking attention in the school environment become a stubborn child at school, often skip school, beat the teacher, cannot stay still in class, do not go to class 2 times, elementary education is not completed, shows the possibility of parenting parents who are overt rejection so have problems with childhood behavior disorders. Judging from social factors, patients have problems with "hemorrhoid" disease that has long been harbored and rarely told.

\section{DIFFERENT DIAGNOSISS}

1. Organic hallucinosis (F06.0)

2. Delirium not due to Alcohol and other Psychoactive (F05)

3. Acute Schizophrenic Psychotic Disorder (F23.2)

\section{MULTIAXIAL EVALUATION}

Axis I : Organic hallucinosis (F06.0) according ICD 10 diagnosis.

Axis II : Narcissistic Personality with Repressive ego defense mechanisms.

Axia III : Hemorrhagic stroke ec thrombus; CHF FC II ec suspend HHD, HT stage II; Dyslipidemia; hyperuricemia; Grade IV internal hemorrhoids.

Axis IV : Problem related to the disease.

Axis V:GAC current F: 30-21 with best GAF last 1 year: 90-81.

Physical: Hemorrhagic stroke ec thrombus; CHF FC II ec suspend HHD, HT stage II; Dyslipidemia; hyperuricemia; Grade IV internal hemorrhoids

Psychologist: Having pain conditions experienced, "hemorrhoids" pain and desire for surgery immediately and Narcissistic personality traits with ego defense mechanisms Repression

Social: having problem with "hemorrhoids" disease that has been hidden for a long time and is rarely told. 


\section{PROGNOSIS}

Quo ad vitam: dubia ad night Quo ad functionm: dubia ad night

Quo ad sanationam: dubia ad night

Profit of prognosis: Complaints of stroke for the first time, patient is cooperative enough to take medication, clear stressor, good family support

Factor aggravating the prognosis: the patient's organic disease, namely: nonhemorrhagic stroke ec thrombus; CHF FC II ec suspec HHD, HT stage II; Dyslipidemia; hyperuricemia; Grade IV internal hemorrhoids, genetic factors present, narcissistic personality traits with ego repression defense mechanisms

\section{Pharmacotherapy}

Psychiatric: Haloperidol 0.75 milligrams every 24 hours intraorally

Neurologic: Mobility in gradually

- infusion Nacl 0.9\% 20 drops per minute

- Citicoline 500 milligrams every 12 hours intraorally

- Ranitidine 150 milligrams every 12 hours intraorally

- Lactulose 10 cc every 8 hours

- Paracetamol 1000 milligrams every 8 hours intraorally

- Simvastatin 40 milligrams every 24 hours intraoral

- Allopurinol 100 milligrams every 24 hours intraorally

- Codeine 10 milligrams every 8 hours intraorally

- Adalat Oros 30 milligrams every 24 hours intraorally

\section{Psychotherapy}

Supportive to patients: provide warmth, empathy, understanding and optimism. Help the patient identify and express emotions and assist with ventilation.

Supportive to family: provide education to the family about the mental condition of the patient is very useful for the management of the disorder suffered. The main purpose of education is to provide an understanding of the illness and appropriate treatment according to the patient's condition. By involving the family in educational sessions, it will be able to provide better support for the patient's recovery.

\section{DISCUSSION}

In ischemic stroke, the neurons are denied of blood and consequently lose a lot of their stock of oxygen and glucose. In a hemorrhagic stroke, the cerebrum is overwhelmed with blood and abundance oxygen, calcium, and different synthetic substances. Both ischemia and drain cause a large number of similar issues, including cerebrum edema, which expands strain on the mind and the probability of extra strokes. Both ischemia and drain additionally debilitate the sodium-potassium siphon, prompting sodium amassing inside neurons. The blend of edema and overabundance sodium triggers an overarrival of the transmitter glutamate, which overwhelms neurons so sodium and different particles enter the neurons quicker than the sodium-potassium siphon can oust them. Abundance positive particles block digestion in mitochondria and kill neurons. At the point when neurons kick the bucket, microglia cells multiply.[7]

CINAHL, MEDLINE and PsychINFO searches of post-stroke psychosis studies distributed somewhere in the range of 1975 and 2016. The commentators autonomously chose reads up for incorporation, extricated information and evaluated concentrate on quality. Of the 2442 references, 76 met the consideration standards. The middle age for post-stroke psychosis was 66.6 years with somewhat a greater number of guys than females impacted. Deferred beginning is incessant. Neurological highlights are common for stroke, yet a critical minority have a 'quiet stroke'. The most well-known psychoses are preposterous issues, trailed by psychoses, for example, schizophrenia and temperament issues with crazy elements. The assessed commonness of dreams was 
$4.67 \%$ (95\% CI $2.30 \%$ to $7.79 \%)$ and hallucination $5.05 \% \quad(95 \%$ CI $1.84 \%$ to 9.65\%). No precise treatment studies were found. Contextual analyses much of the time report reduction of side effects later antipsychotics, yet genuine worries about the underrepresentation of helpless results continue. Injuries are typically correct side of the equator, particularly front facing, fleeting, parietal and right caudate core. As a general rule, post-stroke psychosis is related with poor utilitarian result and high mortality. Psychosis significantly expands the weight of stroke. Postponed beginning addresses a window for early mediation. Research on the wellbeing and adequacy of antipsychotics in this populace is desperately required. parietal and right caudate core. As a rule, post-stroke psychosis is related with poor practical result and high mortality. Psychosis incredibly expands the weight of stroke. Deferred beginning addresses a window for early mediation. Research on the security and adequacy of antipsychotics in this populace is desperately required. parietal and right caudate core. By and large, poststroke psychosis is related with poor practical result and high mortality. Psychosis enormously builds the weight of stroke. Postponed beginning addresses a window for early intercession. Research on the wellbeing and viability of antipsychotics in this populace is critically required.[8]

Brain imaging studies have recognized a few neural organizations and circuits in the mind with changed capacity in patients with schizophrenia. These incorporate the hippocampo-cerebellocortical circuit, the prefrontal-thalamiccerebellar circuit, useful joining in the respective caudate core, and the striking nature network comprising of the separate cortex, foremost parietal cingulate cortex, and striatum, and limbic designs. Credits maniacal indications to any of these organizations in schizophrenia. The attribution can be made with brokenness of one of these organizations with corresponding insane indications. We present the instance of a patient giving newbeginning hallucinations and stroke in cerebrum areas like the striking nature organization (isolated cortex, parietal cortex, and striatum).[7,9]

The most widely recognized enhanced visualization following retrochiasmal pathway sores is homonymous hemianopia $(\mathrm{HH})$, in which every persistent's eye is ignorant concerning the contralesional visual field. According to a conduct point of view, as well as displaying serious impedance in their contralesional visual fields, hemianopic patients may likewise introduce certainly remaining limit, presently typically alluded to all in all as blindsight. It has as of late been shown that $\mathrm{HH}$ patients can likewise experience the ill effects of an unobtrusive shortage in their ipsilesional visual field, called blind vision (inverse blindsight). Besides, the idea of visual disability in the contralesional and ipsilesional planes, just as the example of practical redesign in the occipital projections of $\mathrm{HH}$ patients later stroke, all give off an impression of being subject to the site of the lesio.[9-11]

In this case, it is known that the patient had a homaragic stroke et causa thrombus who had organic hallucinosis. As per the ICD-10, natural hallucinosis is relentless or intermittent hallucinations because of a fundamental natural reason, notwithstanding common principles for the finding of natural mental problems. Messes with a realized natural reason are called natural mental issues. The most widely recognized natural reason is stroke. The most widely recognized kind of hallucinations are visual visualizations. Visual hallucinations happen by physical aggravations in the unimodal affiliation cortex, orbitofrontal cortex, paralimbic cortex, limbic cortex, striatum, thalamus, midbrain.[8,12]

A stroke is brain damage that happens unexpectedly brought about by a disappointment of blood supply where there is a blood coagulation in one of the corridors or veins of the cerebrum that 
blocks dissemination to specific spaces of the mind or a course breaks (drains). The effect of stroke can cause intellectual and perceptual aggravations. The more normal kind of stroke is ischemia, the consequence of a blood coagulation or other block in the corridor. Another uncommon kind is dying, coming about because of blood vessel burst.[13]

\section{CONCLUSION}

We report a case of a 43-year-old male patient who was known to have hallucinatory symptoms with clinical symptoms of acute non-hemorrhagic stroke et causa thrombus in the right hemisphere. Therapy in these patients must be comprehensive by combining psychiatric and neurological disorders

\section{Conflict Of Interest}

The authors declare no conflict of interest, financial or otherwise.

Acknowledgments: declared none.

Patients' Consent: The patient has given permission and informed consent for the publication of this case report.

\section{REFERENCES}

1. Bujarski, K. A. \& Sperling, M. R. Encyclopedia of Human Behavior. 2nd ed. California: Academic Press. 2012

2. Kim NY, Hsu J, Talmasov D, Joutsa J, Soussand $\mathrm{L}, \mathrm{Wu} \mathrm{O}$, et al. Lesions causing hallucinations localize to one common brain network. Mol Psychiatry. 2021;26(4):1299309.

3. Santos H, Agrela P, Parente C, Lopes K, Santos S, Cardoso A, et al. Charles bonnet syndrome as a rare complication in an ischaemic stroke. Eur Neurol Rev. 2018; 13(2):120-2.

4. Chokron S, Perez C, Peyrin C. Behavioral consequences and cortical reorganization in homonymous hemianopia. Front Syst Neurosci. 2016;10(JUN):1-12.
5. Carota A, Bogousslavsky J. Neurology versus Psychiatry? Hallucinations, Delusions, and Confabulations. Front Neurol Neurosci. 2019;44:127-40.

6. Kalat, J. W., 2009. Biological Psychology. 10th ed. North Carolina State: Wadsworth, Cengage Learning. 2009;2009.

7. Nanda S, Priya K, Khan T, Patel P, Azizi H, Nuthalapati D, et al. Combined ParietalInsular- Striatal Cortex Stroke with NewOnset Hallucinations: Supporting the Salience Network Model of Schizophrenia. Psychiatry J. 2020;2020:1-6.

8. Stangeland H, Orgeta V, Bell V. Poststroke psychosis: A systematic review. J Neurol Neurosurg Psychiatry. 2018;89(8):879-85.

9. Martinelli F, Perez C, Caetta F, Obadia M, Savatovsky J, Chokron S. Neuroanatomic correlates of visual hallucinations in poststroke hemianopic patients. Neurology. 2020;94(18):e1885-91.

10. Manabe Y, Kosaka K. Behavioral and psychological symptoms of dementia. Dement with Lewy Bodies Clin Biol Asp. 2016;I:87-92.

11. Rafique SA, Richards JR, Steeves JKE. Altered white matter connectivity associated with visual hallucinations following occipital stroke. Brain Behav. 2018;8(6):110.

12. Morenas-Rodríguez E, Camps-Renom P, Pérez-Cordón A, Horta-Barba A, SimónTalero M, Cortés-Vicente E, et al. Visual hallucinations in patients with acute stroke: a prospective exploratory study. Eur J Neurol. 2017;24(5):734-40.

13. Tini K, Samatra IDPGP, Wiryadana KA, Supadmanaba IGP. Clinical profile of patients with cerebrovascular disease at stroke unit, sanglah general hospital, Denpasar, Bali. Bali Med J. 2020;9(1):12936.

How to cite this article: Pricella Maria Ismail, Anak Ayu Sri Wahyuni, Anak Agung Ayu Suryapraba Indradewi. Organic hallucinosis in non-hemorrhagic stroke caused by thrombosis process: a case report. International Journal of Research and Review. 2022; 9(1): 51-58. DOI: https://doi.org/10.52403/ijrr.20220108 